\title{
FONOLOGÍA DE LA ENTONACIÓN. ASOCIACIÓN PRIMARIA Y SECUNDARIA EN DIALECTOS ANTÍPODAS: ESPAÑOL DE BUENOS AIRES Y DE ESPAÑA
}

\author{
Guillermo Andrés Toledo \\ Conicet, Argentina \\ Université Laval, Canadá
}

\begin{abstract}
Resumen
En el presente trabajo se analiza la influencia de la frase entonativa intermedia (ip), la inflexión ascendente y el acento de frase (H-) en la formalización del acento tonal final de esa ip. El análisis se basa en la Fonología métrica y autosegmental (AM). Los acentos tonales finales se realizan en palabras paroxítonas, proparoxítonas y oxítonas. El corpus de laboratorio está integrado por producciones de hablantes peninsulares (Barcelona y Alicante) y de hablantes argentinos (Buenos Aires). Los acentos tonales finales de ip dentro de ítems paroxítonos y proparoxítonos presentan una estructura formal $\mathrm{L}^{*}+\mathrm{H}$, con posrealización del pico tonal en la sílaba postónica. Opuestamente, los acentos tonales finales de ip dentro de palabras oxítonas muestran una estructura formal $\mathrm{L}+\mathrm{H}^{*}$, con la realización del pico tonal en la sílaba acentuada. Los resultados sugieren una asociación fonológica secundaria entre el tono y el acento de frase (H-).
\end{abstract}

Palabras clave: Fonología métrica y autosegmental (AM), Fraseo entonativo, Asociación fonológica primaria, Asociación fonológica secundaria.

Abstract

The tonal influence of the intermediate phrase (ip), and the edge rising tone (H-) on the preceding final accent $\left(\mathrm{T}^{*}\right)$ is analyzed. The model is Autosegmental Metrical (AM). Two ip phrases are studied: ( $\left.\mathrm{T}^{*} \mathrm{H}-\right)$ (ip), $\mathrm{T}^{*}$ are pitch accents in proparoxytone, paroxytone, and oxytone words emitted by Peninsular and Buenos Aires speakers. The results indicate that $\mathrm{H}$ - in front of $\mathrm{T} *$ causes changes in the phonological association. Proparoxytone and paroxytone T*s show peak delay due to the $\mathrm{H}$ continuation rise on the post-tonic syllable. In oxytones, the peak takes place on the accented syllable and overlaps the $\mathrm{H}$-. The results suggest a secondary phonological association of the last ip $\mathrm{T}^{*}$ with the $\mathrm{H}$ - edge tone.

Key Words: Autosegmental and Metrical Phonology (AM), Spanish Phrasing, Primary Phonological Association, Secondary Phonological Association.

\section{INTRODUCGIÓN}

En un análisis tradicional de la entonación (Navarro Tomás 1968, pp. 208-235), una emisión declarativa de dos grupos fónicos presenta 
una rama tensiva en el primer grupo fónico y una rama distensiva en el segundo grupo fónico. El primer grupo fónico muestra un tonema de semianticadencia de dos a tres semitonos. El segundo grupo fónico muestra un tonema de cadencia de ocho semitonos. La rama tensiva, la prótasis, indica un valor pragmático de continuidad, con un tonema ascendente. La rama distensiva, la apódosis, culmina la emisión con un tonema descendente. Ambos tonemas son perceptivamente significativos y están sobre el umbral perceptivo. Para el español el umbral perceptivo es de 1.50 semitonos (Pamies Bertrán y otros 2002). Sosa 1999, pp. 125-129 analiza los tonemas ascendentes del español de manera similar.

La estructura de este trabajo es la siguiente: en el Apartado I se presenta una introducción al tema. En la Sección 1 se explica el modelo métrico y autosegmental (AM); en la Sección 2 se presentan los acentos tonales de acuerdo a la propuesta de Hualde 2003; en la Sección 3 se muestran los acentos tonales en el español y su variabilidad dialectológica que se basa en los estudios de Sosa 1999; en la Sección 4 se revisan los estudios sobre el español de Buenos Aires y la peculiaridad que lo opone, en líneas generales, al español de la mayoría de los dialectos; en la Sección 5 se revisa el fraseo entonativo del español y las unidades jerárquicas que lo constituyen; en la Sección 6 se estudia la frase entonativa intermedia, se revisan las posiciones opuestas a esta unidad jerárquica y las posiciones que asume esta unidad en sus análisis entonativos; en la Sección 7 se estudia la asociación fonológica secundaria y se la compara con la asociación fonológica primaria, se puntualiza el análisis en la asociación fonológica secundaria en unidades de diferente nivel jerárquico (las sílabas acentuadas y los tonos de frontera); en la Sección 8 se revisan los trabajos sobre asociación fonológica secundaria (en niveles jerárquicos diferentes) en corpus discursivos del español peninsular y en corpus discursivos del español de Buenos Aires. En el Apartado II se presenta la metodología del estudio. En la Sección 1 se consignan los corpus de estudio y los hablantes; en la Sección 2 se explica el análisis acústico de los corpus. En el Apartado 3 se presentan los resultados de los diferentes corpus estudiados. En el Apartado 4 se concluye y se discuten los hallazgos por medio de argumentaciones que confrontan otros trabajos realizados sobre el tema.

\subsection{El modelo métrico y autosegmental}

En el modelo métrico y autosegmental (AM), el contorno de entonación se genera por la suma de acentos tonales asociados a las sílabas acentuadas (Pierrehumbert 1980, Pierrehumbert y Beckman 1988, Ladd 1996, Gussenhoven 2002, Beckman y otros 2002, Beckman y otros 
2005). Estos están asociados a un máximo tonal o a un mínimo tonal y se definen por medio de niveles tonales estáticos, Alto $(\mathrm{H})$ y Bajo (L) (Ladd 1996, p. 43). Estos niveles estáticos están en relación paradigmática y son relativos al rango tonal del hablante (el valor más alto y el valor más bajo), en lugar de serlo en forma sintagmática, esto es, con referencia a los acentos tonales adyacentes (Beckman y otros 2005). La sílaba a la que están asociados esos tonos es central y tiene valor fonológico, los tonos en el contexto silábico adyacente, a derecha y a izquierda, son periféricos y fonéticos (Ladd 1996, pp. 46-47), esto es, son tonos flotantes (Pierrehumbert 1980). En el plano métrico, la sílaba acentuada central es la más fuerte y las sílabas periféricas son más débiles (Pierrehumbert 1980).

\subsection{Los acentos tonales}

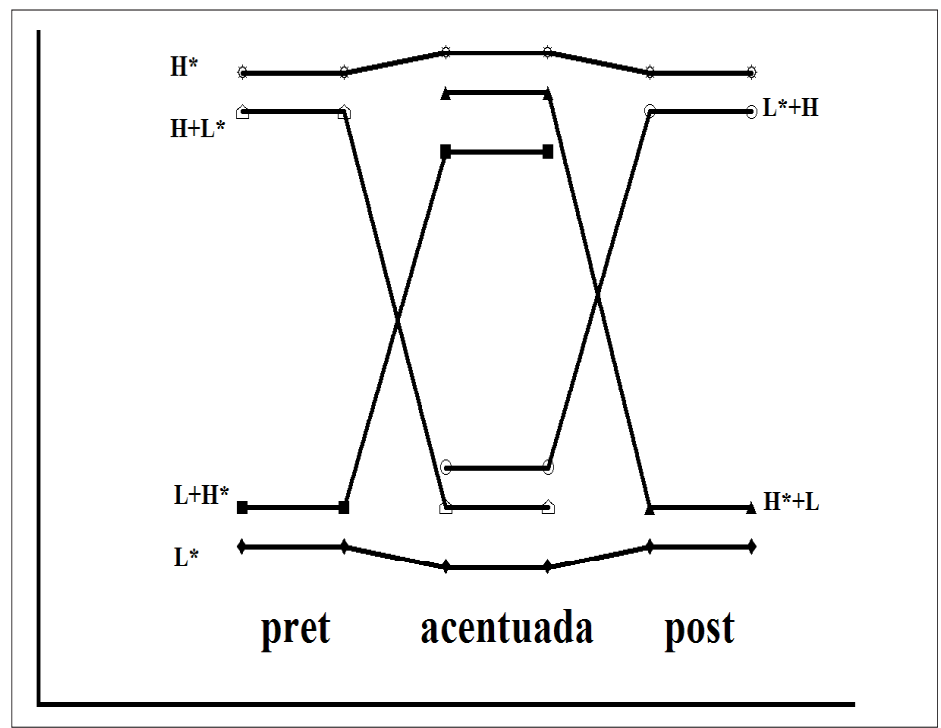

Fig. 1 Acentos tonales

En la Fig. 1 se muestra un esquema de los seis acentos tonales propuestos para el español por Hualde 2003. El primer acento tonal es monotonal, es $\mathrm{H}^{*}$ y muestra un pico en la acentuada, pequeño, sin un valle precedente o siguiente (pret, post). El segundo acento tonal es $\mathrm{H}_{+} \mathrm{L}^{*}$ y presenta un valle en la acentuada precedido por un pico en la pretónica, esto es, un descenso de la pretónica a la acentuada. El tercer acento tonal es $\mathrm{L}+\mathrm{H}^{*}$ : hay un pico en la sílaba acentuada precedido por un valle, es decir, hay un ascenso en la pretónica (pret) a la acentuada. El cuarto acento tonal es también monotonal y es L*. Presenta un valle en la sílaba 
acentuada y no hay movimientos tonales significativos en el contexto de esa sílaba acentuada (pret, post). El quinto acento tonal es $\mathrm{L}^{*}+\mathrm{H}$ y muestra un valle en la acentuada seguido por un pico en la postónica, es decir, se produce un ascenso desde la acentuada hacia la postónica. El sexto es $\mathrm{H}^{*}+\mathrm{L}$ y tiene un pico en la acentuada seguido por un valle en la postónica (post). Se produce un descenso tonal desde la acentuada.

\subsection{El español hispanoamericano y peninsular}

\begin{tabular}{|l|c|c|c|c|}
\hline Buenos Aires & $\mathrm{H}^{*}+\mathrm{L}$ & $\mathrm{H}^{*}$ & $\mathrm{~L}^{*}$ & $\mathrm{~L} \%$ \\
Bogotá & $\mathrm{L}^{*}+\mathrm{H}$ & $\mathrm{L}^{*}+\mathrm{H}$ & $\mathrm{H}^{*}$ & $\mathrm{~L} \%$ \\
México & $\mathrm{L}^{*}+\mathrm{H}$ & $\mathrm{H}^{*}$ & $\mathrm{~L}+\mathrm{H}^{*}$ & $\mathrm{~L} \%$ \\
San Juan & $\mathrm{L}^{*}+\mathrm{H}$ & $\mathrm{L}^{*}+\mathrm{H}$ & $\mathrm{L}^{*}$ & $\mathrm{~L} \%$ \\
Caracas & $\mathrm{L}^{*}+\mathrm{H}$ & $\mathrm{L}^{*}+\mathrm{H}$ & $\mathrm{H}^{*}$ & $\mathrm{~L} \%$ \\
Cuba & $\mathrm{L}^{*}+\mathrm{H}$ & $\mathrm{L}^{*}+\mathrm{H}$ & $\mathrm{L}^{*}$ & $\mathrm{~L} \%$ \\
Sevilla & $\mathrm{L}^{*}+\mathrm{H}$ & $\mathrm{L}^{*}+\mathrm{H}$ & $\mathrm{L}^{*}$ & $\mathrm{~L} \%$ \\
Barcelona & $\mathrm{L}^{*}+\mathrm{H}$ & $\mathrm{L}^{*}+\mathrm{H}$ & $\mathrm{H}^{*}$ & $\mathrm{~L} \%$ \\
Pamplona & $\mathrm{L}^{*}+\mathrm{H}$ & $\mathrm{L}^{*}+\mathrm{H}$ & $\mathrm{L}^{*}$ & $\mathrm{~L} \%$ \\
Madrid & $\mathrm{L}^{*}+\mathrm{H}$ & $\mathrm{L}^{*}+\mathrm{H}$ & $\mathrm{H}^{*}$ & $\mathrm{~L} \%$ \\
\hline
\end{tabular}

Tabla 1. Realizaciones dialectales en hablantes hispanoamericanos y pensinsulares (adaptado de Sosa 1999, pp. 187197) de la emisión declarativa le dieron el número de vuelo

En la tabla 1 se muestran, en adaptación, los resultados indicados por Sosa 1999, pp. 187-197, para el español hispanoamericano y peninsular. La casi totalidad de los dialectos españoles muestran una posrealización del pico tonal en la sílaba postónica. De manera inversa, el español de Buenos Aires muestra acentos prenucleares $\mathrm{H}^{*}$, esto es, el pico se produce en la sílaba acentuada. En la emisión declarativa le dieron el número de vuelo existe un pretonema con dos acentos tonales y un acento nuclear final, más el tono de frontera final $\mathrm{L} \%$. Sosa niega la presencia de frases entonativas intermedias para el español. Los resultados indican que en casi todos los dialectos estudiados los acentos prenucleares son $\mathrm{L}^{*}+\mathrm{H}$. Este acento tonal es el quinto en la Figura 1 (Hualde 2003). Se caracteriza por un valle en la sílaba acentuada seguido por un pico que se despliega en el espacio temporal de la postónica (post), esto es, se produce un ascenso en la sílaba acentuada que culmina en la sílaba postónica. El dialecto de Buenos Aires se aparta abiertamente de este esquema prosódico: presenta un pretonema con acentos tonales en la sílaba acentuada $\left(\mathrm{H}^{*}\right)$, monotonales y bitonales. El dialecto de México presenta una variante: el primer acento prenuclear sigue el esquema de la mayoría de dialectos del español y el segundo acento tonal sigue el esquema de la entonación del español de Buenos Aires. 


\subsection{El español de Buenos Aires}

Barjam 2004 obtiene en el español de Buenos Aires una taxonomía compuesta por dos tonos fonemáticos prenucleares con la asociación fonológica dentro de la sílaba acentuada: $/ \mathrm{L}+\mathrm{H}^{*} / \mathrm{y} / \mathrm{L}+\wedge \mathrm{H}^{*} /$. El diacrítico del segundo acento tonal indica un efecto de upstep 'escalonamiento ascendente'. Los resultados coinciden, en líneas generales, con los de Colantoni y Gurlekian 2004 y Colantoni 2005 para el dialecto de Buenos Aires. Colantoni y Gurlekian 2004 estudian un corpus de 1200 oraciones declarativas. En sus resultados, un $90 \%$ de los acentos prenucleares presentan la mayor prominencia en la sílaba acentuada. Colantoni 2005 analiza también los acentos prenucleares en corpus de Argentina. Sus hallazgos indican acentos $\mathrm{H}^{*}+\mathrm{L}$, con una frecuencia de aparición relativa de $90.74 \%$ y acentos $\mathrm{L}+\mathrm{H}^{*}$, con una frecuencia de aparición relativa de $1.85 \%$. Los fenómenos de posrealización del pico sobre la sílaba postónica son rarísimos en ese corpus, como lo muestra Sosa 1999, p. 187 y Toledo 2000.

\subsection{El fraseo entonativo}

Las emisiones entonativas se estructuran en niveles jerárquicos. El nivel de mayor importancia es la frase entonativa mayor (IP). Esta unidad está integrada por varias frases entonativas intermedias (ip). La frase entonativa intermedia tiene por lo menos un acento tonal. Dentro de ella se encuentran las palabras (w) y las sílabas. La frase entonativa mayor y la frase entonativa menor tienen tonos de frontera que marcan fonológicamente estas unidades. La IP se cierra por medio del tono L\% о $\mathrm{H} \%$, bajo o alto. La ip se cierra por medio del tono L- $\mathrm{o} \mathrm{H}^{-}$. En general, la ip indica pragmáticamente una continuidad textual o discursiva. Por lo tanto, esta marca se produce por medio de un tono H-, alto. En corpus de laboratorio, por lectura, la frase intermedia es generalmente $\mathrm{H}_{-}$. En discursos espontáneos, aparecen tonos de frontera de la ip L- y H-. La regularidad es menos evidente.

La frase entonativa intermedia pertenece al ámbito de la fonología entonativa, no debe confundirse con la frase fonológica $(\phi)$, es decir, una unidad perteneciente a la fonología prosódica. Prieto 2006 analiza coherentemente el fraseo prosódico en español y las $\phi$. En cambio, la ip es una unidad que puede contener un número mayor de ítems que la $\phi$. Esta última está regida por procesos de transformación de la estructura sintáctica en estructura prosódica remitidos siempre a la noción de sintagma. En suma, en este análisis se estudia la frase entonativa intermedia en el ámbito de la fonología entonativa (ver una descripción en Martínez Celdrán y Fernández Planas 2007, pp. 217-219). 
Por otra parte, esta frase entonativa intermedia (ip) es similar al grupo fónico propuesto por Navarro Tomás 1968, p. 217. Como ya ha sido indicado (en el apartado 1.1.), en una oración afirmativa de dos grupos fónicos, el primer grupo fónico presenta una inflexión ascendente y el segundo grupo fónico culmina con una inflexión descendente. Entre ambas estructuras prosódicas existe un cambio tonal manifiesto: cambia de un nivel agudo en el nivel de la primera unidad a un nivel grave en el inicio de la segunda unidad. Pueden producirse pausas entre los grupos fónicos. Los dos grupos fónicos constituyen una unidad que se asemeja a la frase entonativa mayor (IP). En un análisis de niveles, Quilis 1975 propone tres fonemas tonales (1, bajo; 2, medio; 3, alto). El alótono de 1 se produce antes de la juntura terminal descendente $/ \downarrow /$; el alótono 2 se produce en contextos alejados de la juntura terminal; el alótono 3 se produce en el contexto de la juntura terminal ascendente $/ \uparrow /$. Los fonemas de juntura terminal indicados tienen alojunturas. El fonema de juntura terminal descendente tiene dos alojunturas: descendente baja, que se actualiza en el final del enunciado y descendente, que se actualiza en posiciones no finales de enunciado. En simetría, el fonema de juntura terminal ascendente tiene dos alojunturas: ascendente alta, que se observa en el final del enunciado y ascendente, que se observa en posiciones no finales del enunciado. Se observa claramente la influencia de las junturas terminales sobre los fonemas tonales, esto es, la influencia de las alojunturas sobre los alótonos, tanto en posiciones intermedias como en posiciones finales.

\subsection{La frase entonativa intermedia en español}

Se discute la importancia de la frase entonativa intermedia del español. Jun 2005 considera que la ip podría no ser relevante para el español. En realidad, se cuestiona su existencia, pero no se niega. Beckman y otros 2002 no aceptan esta unidad, sino tan solo la presencia de la frase entonativa mayor (IP). De manera similar, Sosa 1999 propone que la única unidad entonativa es la IP: el hablante reestructura las emisiones y abre nuevas IP por razones pragmáticas: focos contrastivos y énfasis. Sosa 1999, p. 43 indica estos casos de reestructuración: [nos contaron la triste historia de un ranchero enamorado] IP, [nos contaron] IP [la triste historia de un ranchero enamorado] IP, [nos contaron] IP [la triste historia] IP [de un ranchero enamorado]. Opuestamente, para Nibert 2000 esta es una unidad entonativa de suma importancia, entre otras causas, para evitar la ambigüedad semántica de algunas emisiones (vimos pueblos) ip H- (y campos abandonados) ip o (vimos pueblos y campos abandonados) ip T-, ver Hualde 2003, p. 13. Hualde 2002, 2003 coincide con Nibert en este aspecto, aunque no considera que el acento final de la ip sea de naturaleza nuclear. No diferencia los acentos prenucleares que integran la ip y el acento final antes del tono de frontera (T-).

Nibert 2000, p. 70 propone cuatro pistas prosódicas para la ip: (1) un ascenso tonal en la frontera derecha de la ip: este incremento no 
está asociado a ningún acento tonal, sino a la estructura de la ip; (2) un alargamiento de la sílaba final; (3) un índice de corte de nivel 3, según el ToBI, una pausa o una ruptura perceptible; (4) un cambio tonal después de la ip, ya sea una caída considerable o un contorno aplanado.

En la figura 2 se observa la oración declarativa Las bananas estaban en el armario (hablante femenino, peninsular, Alicante). Se observa claramente la rama tensiva y el movimiento ascendente provocado por el tono de frontera (ver el acento quinto en la figura 1 y Hualde 2003). En lan figura 3 se indica el contorno entonativo de la oración declarativa Las casonas estaban en la colina (hablante femenino, peninsular, Barcelona). Se puede observar el cambio tonal en la apódosis (la rama distensiva), después del ascenso tonal provocado por el tono de frontera de la primera ip, la prótasis (la rama tensiva). En este caso, se produce una caída considerable.

\subsection{La asociación fonológica secundaria}

La asociación fonológica primaria asocia los tonos a las sílabas acentuadas para determinar una taxonomía tonal en contraste lingüístico. En esa asociación, la información autosegmental propone la forma de los acentos tonales: no hay una relación estricta entre la alineación fonética y el tono, antes o después de la sílaba acentuada.

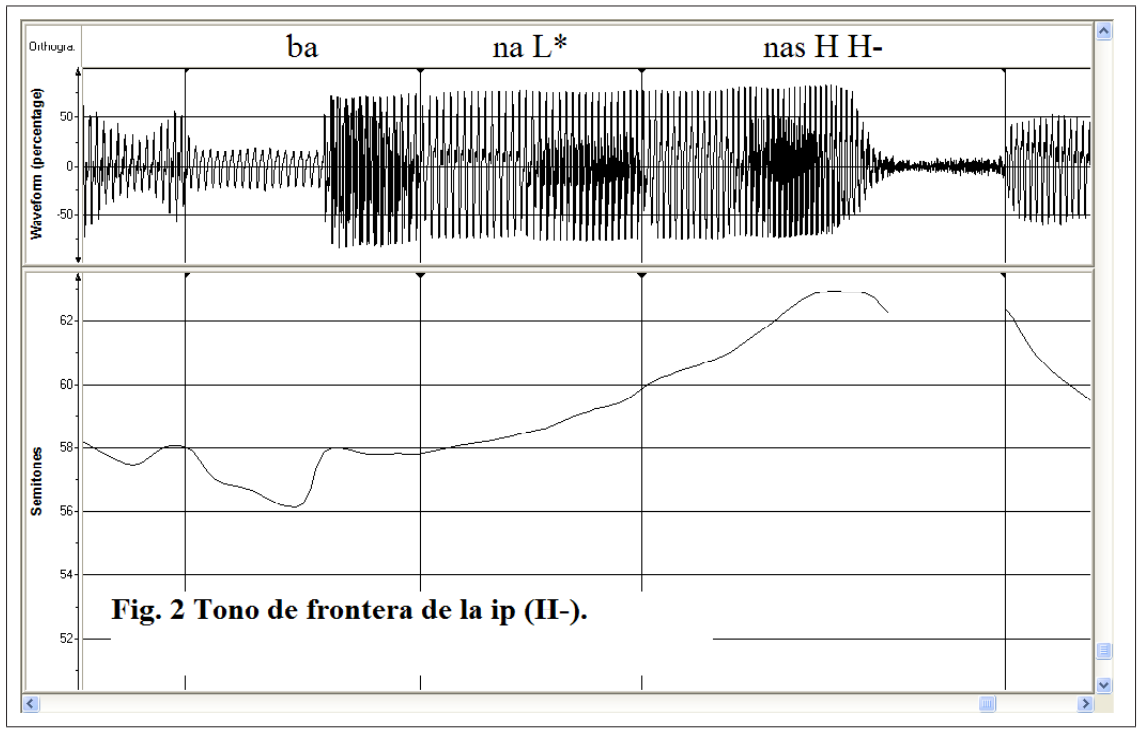

En cambio, la información métrica marca la percepción fuerte o débil de la sílaba y el tono marcado por el diacrítico asterisco (*). Esta relación es perceptiva y no indica tampoco una estricta relación entre lo fonético y lo fonológico (ver para el español y el catalán Prieto 2005). 
La asociación secundaria presenta una asociación fonológica de un tono a dos constituyentes entonativos de nivel jerárquico diferente, por ejemplo, el tono asociado a una sílaba acentuada y a una frase entonativa intermedia. La asociación secundaria se propone como recurso para solucionar problemas fonológicos que difieren de la asociación primaria y estándar. En los estudios pioneros, se asocian los tonos a las sílabas acentuadas y a las fronteras, esto es, en dos niveles jerárquicos (Pierrehumbert y Beckman 1988). Esta se basa también en el estudio de Grice 1995 sobre el italiano de Palermo. Así, se propone también que ciertas sílabas acentuadas se asocian, en forma secundaria, con tonos de frontera intermedia (ip).

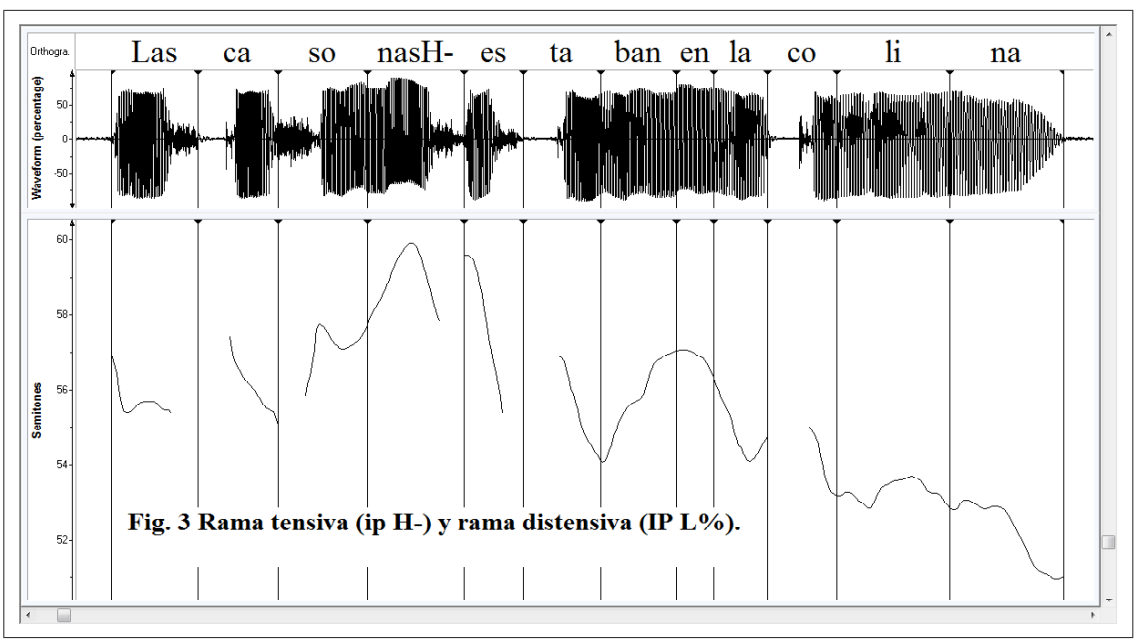

En estudios posteriores, este recurso fonológico se hace extensivo a asociaciones de tonos en el interior de los acentos tonales, esto es, se producen asociaciones secundarias dentro del mismo nivel jerárquico, por ejemplo, en la sílaba acentuada. De esta manera lo usa Prieto 2005 y Prieto y otros 2005 para analizar los tres contrastes en acentos prenucleares del catalán: (1) en el foco estrecho, el tono $\mathrm{H}^{*}$ se asocia con el límite final de la sílaba acentuada; (2) en el foco ancho, el tono H se realiza en la duración de la sílaba postónica; 3) en emisiones interrogativas absolutas, el tono н comienza en la sílaba postónica. En Prieto y otros 2005, el concepto se utiliza en forma similar para estudiar también los contrastes de acentos tonales en napolitano y en el dialecto de Pisa, además del catalán. Asimismo, se propone este recurso fonológico para el dialecto madrileño con el propósito de revisar y completar la terminología estándar propuesta por el Sp-ToBI (los acentos tonales y los tonos de frontera del español, ver Beckman y otros 2002) y la revisión (Face 2006, Face y Prieto 2007). Sin embargo, la asociación fonológica secundaria dentro de un mismo nivel jerárquico (en la sílaba acentuada) no 
es el motivo de la investigación en este artículo; en cambio, sí lo es la asociación fonológica secundaria en niveles jerárquicos diferentes (las sílabas acentuadas asociadas también con tonos de frontera).

En síntesis, la asociación primaria se refiere a la asociación autosegmental del tono con la sílaba acentuada: es la forma de los acentos tonales. No existe una relación estricta entre la asignación fonética y la asociación fonológica. El diacrítico asterisco tiene un valor relativo con respecto a la asignación del tono en la sílaba acentuada. En realidad, indica el tono fuerte o central, el otro tono es débil o periférico. Ese asterisco muestra la percepción de ese tono, con una altura tonal alta o una altura tonal baja. En cambio, la asociación secundaria, en sentido estricto, se refiere a cómo una unidad (en este caso, la sílaba acentuada) y otra unidad de diferente nivel jerárquico (por ejemplo, los tonos de frontera) se vinculan fonológicamente. Esa asociación debe provocar cambios estructurales en los acentos tonales que sean significativos con respecto a otros acentos tonales vinculados en contraste lingüístico (Pierrehumbert y Beckman 1988; Grice 1995). En un sentido más amplio, la asociación secundaria asocia tonos dentro de acentos tonales, en un mismo nivel jerárquico, en el nivel de la sílaba acentuada (para el catalán: Prieto 2005; para el catalán, el napolitano y el italiano de Pisa: Prieto y otros 2005; para el español madrileño: Face 2006; Face y Prieto 2007). Como ya se ha indicado, esto no es parte de este estudio.

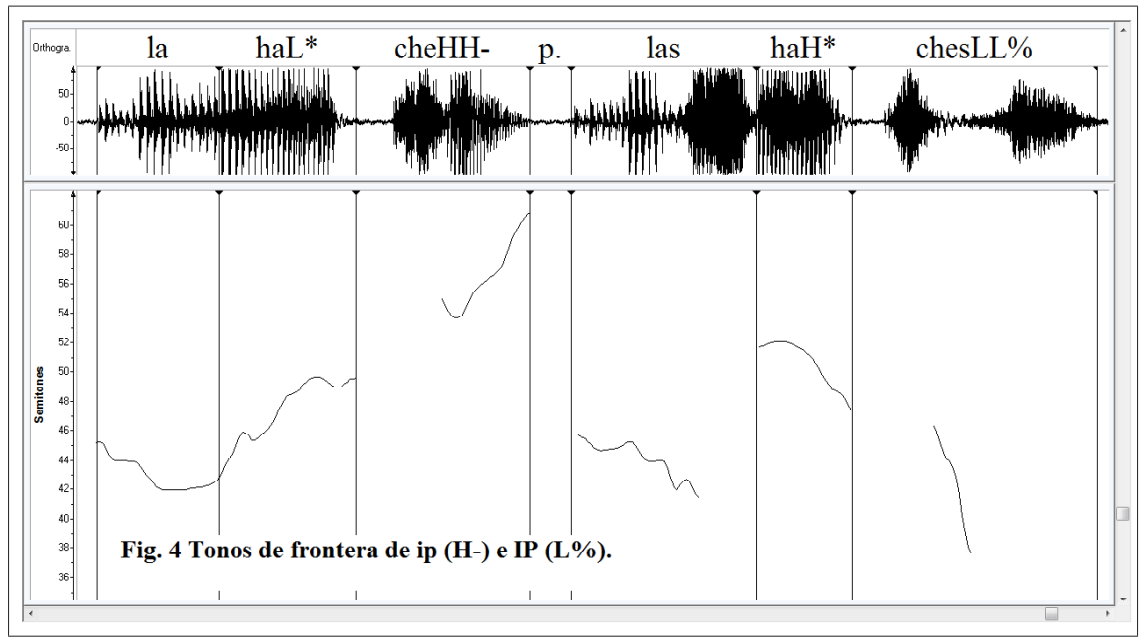

\subsection{Los estudios previos sobre la ip}

En dos estudios anteriores, se analizan las frases entonativas intermedias en dos dialectos antípodas en cuanto a la asociación fonológica primaria y la taxonomía tonal: el dialecto de Buenos Aires (Toledo 2006) 
y el español peninsular, el dialecto de Madrid (Toledo 2007). El primer dialecto, el español de Buenos Aires, no presenta acentos prenucleares en emisiones de foco ancho con pico retrasado en la sílaba postónica, los acentos tonales son $\mathrm{H}^{*}$ y combinados en forma bitonal, por ejemplo, $\mathrm{H}^{*}+\mathrm{L}$ (ver tabla 1 ). El estudio se realiza en un discurso semiespontáneo (Toledo 2006). El segundo dialecto (el madrileño) presenta acentos totales similares al español de la mayoría de los dialectos, es decir, acentos prenuclares en emisiones de foco ancho con picos retrasados, actualizados en la sílaba postónica $\left(\mathrm{L}^{*}+\mathrm{H}\right)$. El análisis se realiza también en discursos semiespontáneos (Toledo 2007). En la figura 4 se presentan dos frases entonativas. El dialecto es peninsular (el español de Madrid). En la frase entonativa intermedia $\left(\mathrm{H}^{-}\right)$, antes de la pausa (p.), se observa el comportamiento tonal de ascenso provocado por el tono de frontera. Asimismo, se puede observar el acento tonal final $\left(\mathrm{L}^{*}+\mathrm{H}\right)$ (ver el quinto acento tonal en la figura 1). En la frase entonativa final (tono de frontera $\mathrm{L} \%$ ), se consigna un ítem similar (las haches), pero el acento tonal en la palabra paroxítona está influido por el tono de frontera descendente $\mathrm{L} \%$, es $\mathrm{H}^{*}+\mathrm{L}$ (ver el sexto acento tonal en la figura 1 ). Los dos acentos tonales tienen asociaciones fonológicas diferentes de acuerdo a la proximidad de tonos de frontera disímiles, uno ascendente y otro descendente (ip H-, IP L\%) (adaptado de Toledo 2006).

En la figura 5 se muestran dos ip: la primera tiene un acento tonal en un ítem paroxítono (miseria, ip $\mathrm{H}_{-}$) y la segunda tiene un acento tonal en un ítem proparoxítono (lágrimas, ip L-). Las emisiones son un fragmento de un relato de vida, un discurso semiespontáneo, producido por un hablante masculino culto, en el dialecto de Buenos Aires. En principio, se observa el movimiento tonal ascendente y descendente en las dos ip.

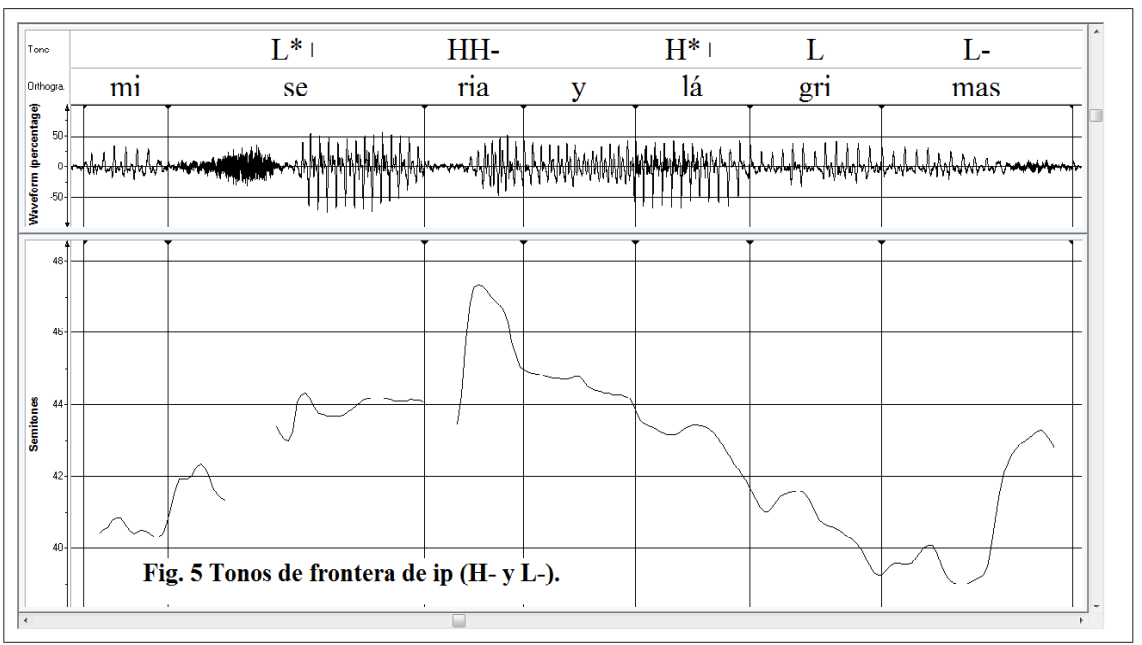


Los dos acentos tonales sufren la influencia de los tonos de frontera. Son $\mathrm{L}^{*}+\mathrm{H}$ Y $\mathrm{H}^{*}+\mathrm{L}$, respectivamente (ver el acento tonal quinto y el acento tonal sexto en la figura 1, adaptado de Toledo 2006). Se observa que la sílaba pretónica en el segundo acento tonal, ligeramente más alta que la sílaba acentuada, se considera una parte del contorno transicional y fonético, sin valor fonológico. El acento tonal, entonces, es $\mathrm{H}^{*}+\mathrm{L}$, resuelto dentro del ítem proparoxítono (lágrimas), no $\mathrm{H}+\mathrm{L} *$ si se tuviera en cuenta el descenso a la sílaba acentuada (y lágrimas). Estos resultados son coincidentes, en líneas generales, con los hallazgos obtenidos en otro estudio sobre el español peninsular, en los Corpus de Referencia del Español Actual (CREA, Universidad Autónoma de Madrid, Real Academia Española), en una muestra de discursos científicos (Toledo, 2008).

En suma, en este nuevo estudio se analizan los acentos tonales en palabras paroxítonas, proparoxítonas y oxítonas en el contexto de la frase entonativa intermedia y con un tono de frontera ascendente $\left(\mathrm{H}^{-}\right)$, esto es, en el contexto pragmático de continuidad semántica. Este contexto es la rama tensiva de la prótasis. Se estudia en un corpus de laboratorio. Se oponen dos dialectos españoles de tipología entonativa opuesta: el español peninsular (acentos prenucleares de pico retrasado) y el dialecto de Buenos Aires (acentos prenucleares sin pico retrasado). Se intenta confirmar lo obtenido en una serie de trabajos sobre discursos semiespontáneos.

\section{Metodología}

\subsection{Los corpus y los hablantes}

Se analizan cuatro corpus de laboratorio. El primer corpus está integrado por diez oraciones declarativas con acentos tonales en palabras paroxítonas en el lado derecho de la frase entonativa intermedia (SN) ip (sv) IP (Amar Roma) (fue inevitable). Los dos acentos tonales están en choque acentual. El segundo corpus presenta frases entonativas intermedias con acentos tonales en items paroxítonos, proparoxítonos y oxítonos: (Pechugas de pavo) ip (con ensalada de coliflor), (Timbal de sémola) ip (aromatizado con roquefort), (Tarta de limón) ip (con nisperos caramelizados). En el tercer corpus se estudian 24 oraciones declarativas (SN) ip (sv). El SN está integrado por un acento tonal en un item paroxítono, en un item proparoxítono o en un ítem oxítono: (Las casonas) ip (estaban en la colina), (Pídele el tónico) ip (al médico), (El camión) ip (se despeñó sobre Gijón). En el corpus 4 se estudia una serie de veinticuatro oraciones similares a las analizadas en el corpus 3, pero se produce una expansión en el SN: (Las casonas marinas) ip (estaban en la colina), (Pídele el tónico mágico) ip (al 
médico), (El camión marrón) ip (se despeñó sobre Gijón). El corpus completo está indicado en el Apéndice. Los hablantes repitieron las emisiones dos veces. Se registran las emisiones de tres hablantes peninsulares (dos hablantes masculinos y un hablante femenino viven en Barcelona, un hablante femenino es oriundo de Alicante). Se registran también las emisiones de dos hablantes femeninos y de un hablante masculino, todos oriundos de Buenos Aires. Las producciones se graban dentro de una cámara silente en condiciones controladas. Los hablantes producen los diferentes corpus y las repeticiones con pausas, en diferentes sesiones de grabación. Todos los hablantes son graduados universitarios. Las producciones peninsulares se llevan a cabo en el Laboratorio de Fonética de la Universidad de Barcelona. Algunos materiales de esta investigación se presentaron en un póster (Toledo y Ramírez Verdugo 2007).

\subsection{Análisis acústico}

Se realizan los contornos de entonación por medio del programa de computación Speech Analizer 3.0 (2007), un analizador del Summer Institute of Linguistics. Las figuras se llevan a cabo por medio del mismo analizador. Se presenta el oscilograma con la forma de onda en la parte superior de la figura y el contorno de entonación en la parte inferior de la figura. Las barras verticales segmentan las sílabas. El etiquetado es ortográfico y se adjuntan los tonos de frontera y el asterisco que indica la sílaba acentuada. En la parte inferior, el eje vertical está segmentado en bandas de dos semitonos, un valor estándar en el programa de computación. Se utiliza la unidad en semitonos porque permite la comprobación de las diferencias de prominencia tonal basada en criterios perceptivos.

\section{LAS FRASES ENTONATIVAS INTERMEDIAS}

En la figura 6 se pueden observar dos frases entonativas intermedias -(Amar Roma) ip (fue inevitable)- ip IP en una emisión producida por un hablante femenino peninsular, dialecto de Barcelona. Las dos ip constituyen una IP L\%. En este caso, cada frase entonativa intermedia coincide con una frase fonológica $(\phi)$. Prieto 2006 analiza las frases fonológicas según los planteos de la Teoría de Optimalidad. Observa un efecto de alignment 'alineamiento': el sujeto se agrupa en una $\phi$ cuando la frase predicado es breve, con una o dos w, por ejemplo (El Presidente de la Generalitat) $\phi$ (sufre) o (Javier) $\phi$ (visitó Galicia) $\phi$. Sin embargo, como se explica arriba, pueden coincidir las unidades o no hacerlo. Prieto propone un índice de corte de la $\phi$ con nivel 2 y según el inventario del 
ToBI. Asimismo, constata una percepción prominente del acento final de esa $\phi$. Este acento no tiene valor nuclear, o por lo menos no está especificado como tal. El índice de corte de la ip (según el ToBi) es de nivel 3 , más definido acústica y perceptivamente.

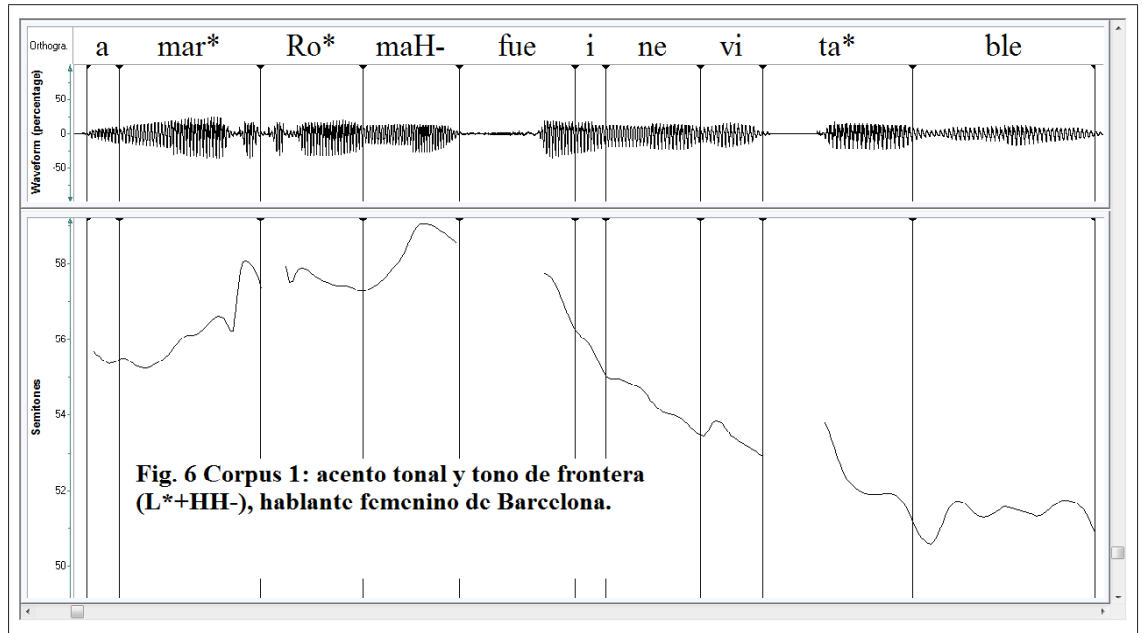

Los dos primeros acentos tonales que constituyen la ip están en posición de choque acentual. Sin embargo, este contexto prosódico no afecta el problema analizado. Prieto 2003 indica que los valores de altura tonal son estables en los dos acentos tonales. Esto es válido tanto para el español como para el catalán. La primera ip (la rama tensiva, la prótasis) presenta una inflexión ascendente debida a la presencia del tono de frontera $(\mathrm{H}-)$. La segunda ip (la rama distensiva, la apódosis) presenta un cambio tonal muy claro: se produce un descenso hacia el tono de frontera final $(\mathrm{L} \%)$. En la primera ip, la relación de los dos acentos tonales no es de downstep 'escalonamiento descendente' como se esperaría en dos acentos tonales del pretonema. En cambio, existe una inflexión ascendente del segundo acento tonal con referencia al primer acento tonal. Este efecto prosódico se debe, sin duda, a la presencia de la frontera de una unidad jerárquica, la ip. Con referencia a la asociación primaria entre la sílaba acentuada y el tono, esta sílaba acentuada es L* y la sílaba postónica presenta un tono H. Esto se produce debido a que la sílaba postónica, en un ítem paroxítono, se incrusta en el tono de frontera. Esta asociación fonológica con una unidad de jerarquía superior con respecto a la sílaba acentuada (es decir: la ip), es un fenómeno de asociación fonológica secundaria (Grice 1995). Sin embargo, podría pensarse que la causa de ello es que los dialectos del español (casi todos, excepto el español de Buenos Aires) presentan acentos prenucleares en emisiones de foco ancho del tipo $\mathrm{L}^{*}+\mathrm{H}$, con pico 
retrasado y realizado en la sílaba postónica (Sosa 1999, Hualde 2002, Face 2001, Hualde 2003).

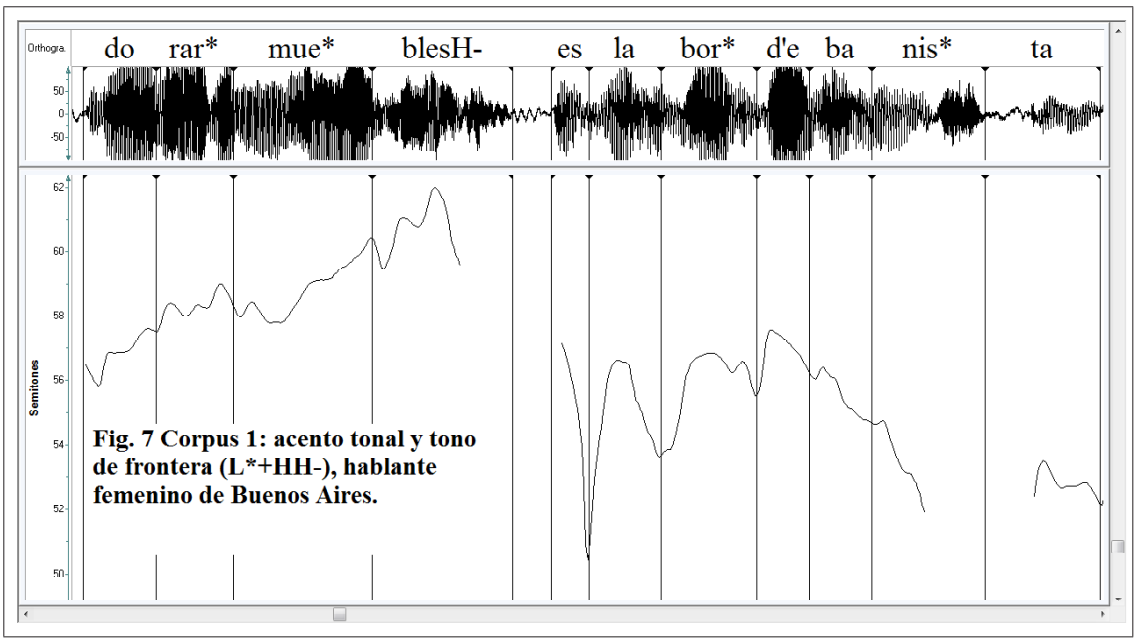

En la figura 7 se observa una emisión declarativa integrada por dos ip -(Dorar muebles) ip (es labor de ebanista) - ip IP. Es una emisión producida por un hablante femenino de Buenos Aires. En principio, este dialecto no comparte las pautas de asociación fonológica del español en general. Los acentos prenucleares en emisiones de foco ancho no presentan un pico retrasado: son acentos tonales $\mathrm{H}^{*} \mathrm{o}$ en combinaciones bitonales $\left(\mathrm{H}^{*}+\mathrm{L}\right)$ (Sosa 1999, p. 187). Sin embargo, los patrones entonativos que se observan son similares a los restantes dialectos del español. Los acentos tonales de la primera ip (la rama tensiva, la prótasis) no presentan escalonamiento descendente entre el primer acento tonal y el segundo acento tonal. Opuestamente, el segundo acento tonal presenta un efecto de escalonamiento ascendente. El efecto se produce por la presencia del tono de frontera que cierra la ip $\left(\mathrm{H}_{-}^{-}\right)$. La segunda ip (la rama distensiva, la apódosis) muestra un cambio tonal considerable, una caída hacia el final de la IP ( $\mathrm{L} \%)$. La asociación fonológica sigue la tendencia mostrada en el caso presentado (la emisión del hablante femenino de Barcelona): una asociación primaria del tono con la sílaba acentuada $\left(\mathrm{L}^{*}\right)$ y un tono $\mathrm{H}$ en la sílaba postónica debido a que esta postónica se incrusta en el espacio tonal reservado al tono de frontera $\mathrm{H}-$. En suma, en este contexto, los hablantes de dos dialectos opuestos (dialecto con pico retrasado y dialecto sin pico retrasado) tienen acentos tonales similares en ítems paroxítonos, es decir, $\mathrm{L}^{*}+\mathrm{H}$ (ver el acento tonal quinto en la figura 1) debido a la asociación fonológica secundaria con el tono de frontera ascendente $(\mathrm{H}-)$. 


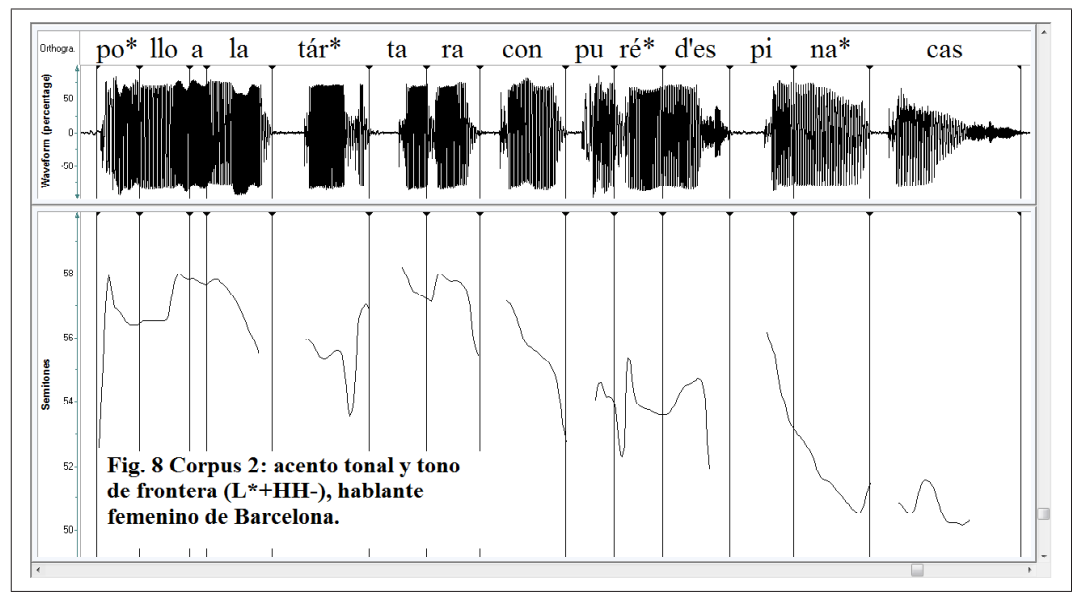

En la figura 8 se presenta una emisión declarativa integrada por dos ip -(Pollo a la tártara) ip (con puré de espinacas)- ip IP. La emisión pertenece al Corpus 2 (ver Apéndice). Es una emisión producida por un hablante femenino de Barcelona. La primera ip tiene un acento tonal final dentro de un ítem proparoxítono (tártara). El esquema entonativo es similar a los ejemplos anteriores. Se produce un ascenso en la rama tensiva debido a la presencia de un tono de frontera $\mathrm{H}_{-} \mathrm{y}$ un cambio tonal descendente en la rama distensiva. El acento tonal final de la primera ip es $\mathrm{L}^{*}+\mathrm{H}$ (ver este acento tonal en la figura 1, es el quinto), con una asociación primaria entre la sílaba acentuada y el tono $\left(\mathrm{L}^{*}\right)$ y una asociación secundaria entre dos unidades de diferente jerarquía (ip H), la postónica presenta un tono periférico н debido a la influencia del tono de frontera $(\mathrm{H}-)$. La segunda sílaba postónica de la palabra proparoxítona mantiene un nivel tonal similar a la primera sílaba postónica.

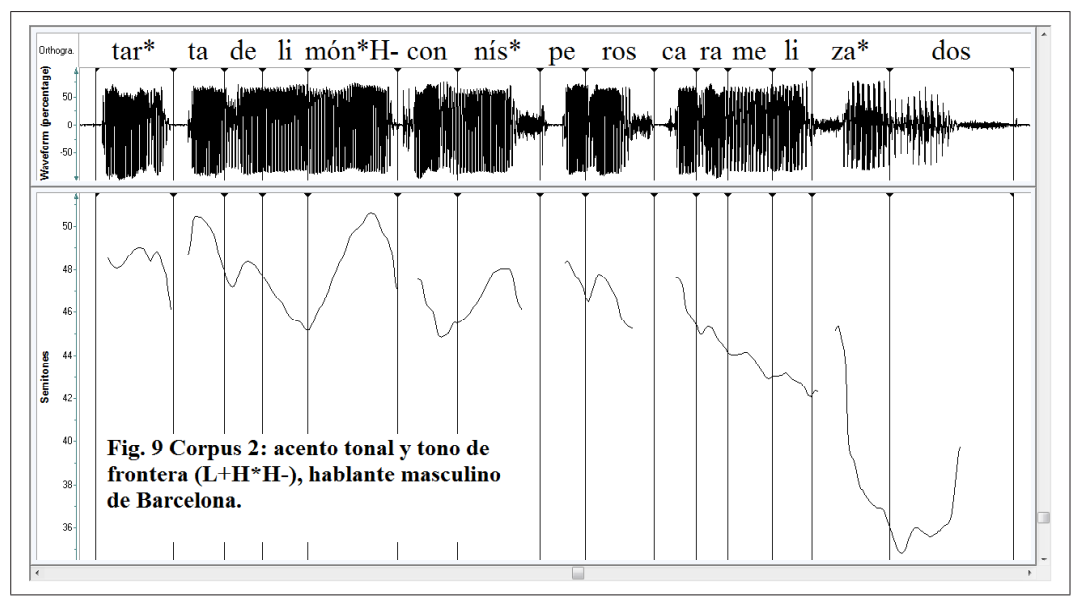


En la figura 9 se presentan dos ip dentro de una emisión declarativa (Corpus 2). La emisión es (Tarta de limón) ip (con nísperos caramelizados) ip IP. Es una producción de un hablante masculino de Barcelona. La entonación es similar a los casos ya presentados. Las dos ip se desarrollan en diferentes rangos tonales: la rama tensiva presenta un crecimiento tonal y la rama distensiva muestra un descenso tonal significativo. El corte se produce siempre después de la cima provocada por el tono de frontera H-. Así, los dos acentos tonales iniciales de la primera ip no muestran un escalonamiento descendente, sino por el contrario, uno ascendente. El primer acento tonal se produce en una palabra paroxítona y es L ${ }^{*}+\mathrm{H}$ (ver el acento tonal quinto en la figura 1). Sigue el esquema de los acentos tonales prenucleares en el español. Sin embargo, el segundo acento tonal, final de la ip, es $\mathrm{L}+\mathrm{H}^{*}$ (ver el acento tonal tercero en la figura 1). Se produce una asociación primaria con la sílaba acentuada y una asociación secundaria con una unidad de estructura jerárquica mayor, la ip. La influencia del tono de frontera y el contexto oxítono del ítem provoca una asociación in situ, en la sílaba acentuada.

En una serie de estudios recientes (de la Mota 2005, Estebas Vilaplana 2006, 2007), se propone que el desplazamiento del pico tonal es relativo al espacio postónico: a mayor número de sílabas postónicas, mayor es la distancia de realización del pico tonal. Los acentos tonales en palabras proparoxítonas presentan un pico más retrasado porque tienen suficiente espacio para realizarse. Opuestamente, los acentos tonales en palabras oxítonas no tienen espacio postónico, y por lo tanto, el pico se produce más adelantado. El efecto es de truncamiento en los acentos tonales de palabras oxítonas. Esta explicación podría ser coherente en producciones de dialectos con pico retrasado, por ejemplo, este caso, en el dialecto de Barcelona. Asimismo, en la mayoría de dialectos del español (Sosa 1999; Hualde 2002; Face 2001; Hualde 2003). Se observa, además, el comportamiento opuesto de los acentos tonales en palabras proparoxítonas (ascendente en la figura 8; descendente en la figura 9, lo cual viene provocado por diferentes contextos: rama tensiva y rama distensiva, respectivamente. Estebas Vilaplana y Prieto 2007, sin embargo, observan en un test de producción que el pico de F0 se alinea después de la sílaba acentuada en acentos tonales dentro de los tres tipos de palabras: paroxítonas, proparoxítonas y oxítonas. El efecto de truncamiento en acentos tonales en palabras oxítonas quedaría anulado. 


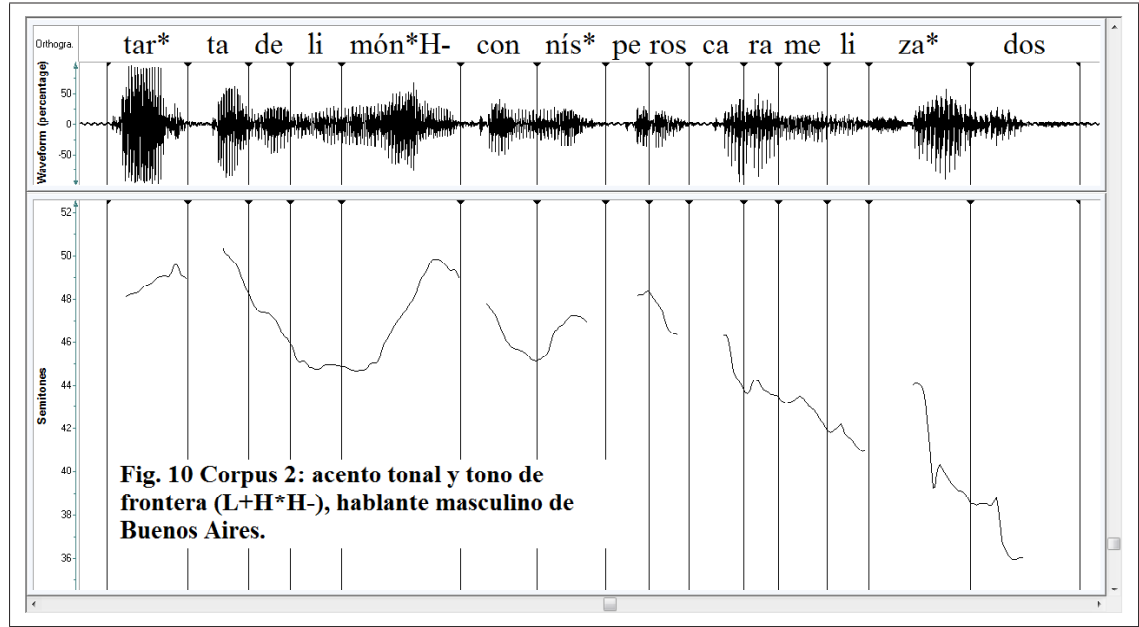

En la figura 10 se presenta la entonación en una emisión similar a la figura 9: dos ip dentro de una emisión declarativa. Es una producción de un hablante masculino de Buenos Aires. Los dos acentos tonales de la primera ip no muestran un escalonamiento descendente. En realidad, el segundo acento tonal está influido por el tono de frontera ascendente, lo que impide el escalonamiento descendente. Las dos ip (la de la rama tensiva y la de la rama distensiva) se desarrollan en diferentes rangos tonales. La primera ip está sostenida por el tono de frontera y la segunda ip decrece considerablemente hasta el tono de frontera final ( $\mathrm{L} \%$ ). La taxonomía del acento tonal es similar a la registrada en la figura 9 , un acento tonal $\mathrm{L}+\mathrm{H}^{*}$. Se producen las dos asociaciones, la primaria y la secundaria, las dos in situ, en la sílaba acentuada (ver el acento tonal tercero en la figura 1). En suma, en un dialecto con acentos tonales que no presentan picos retrasados, también se produce una tendencia entonativa similar a la registrada en un dialecto con acentos tonales que muestran picos retrasados (un dialecto con acento tonales $\mathrm{H}^{*}+\mathrm{L}$ y un dialecto con acentos tonales $\mathrm{L}^{*}+\mathrm{H}$, respectivamente). Este contraargumento se opone al argumento anterior sobre el truncamiento en acentos tonales de palabras oxítonas (ver de la Mota 2005; Estebas Vilaplana 2006). En la rama distensiva, el acento tonal del ítem proparoxítono (nísperos) desciende desde la segunda sílaba postónica. 


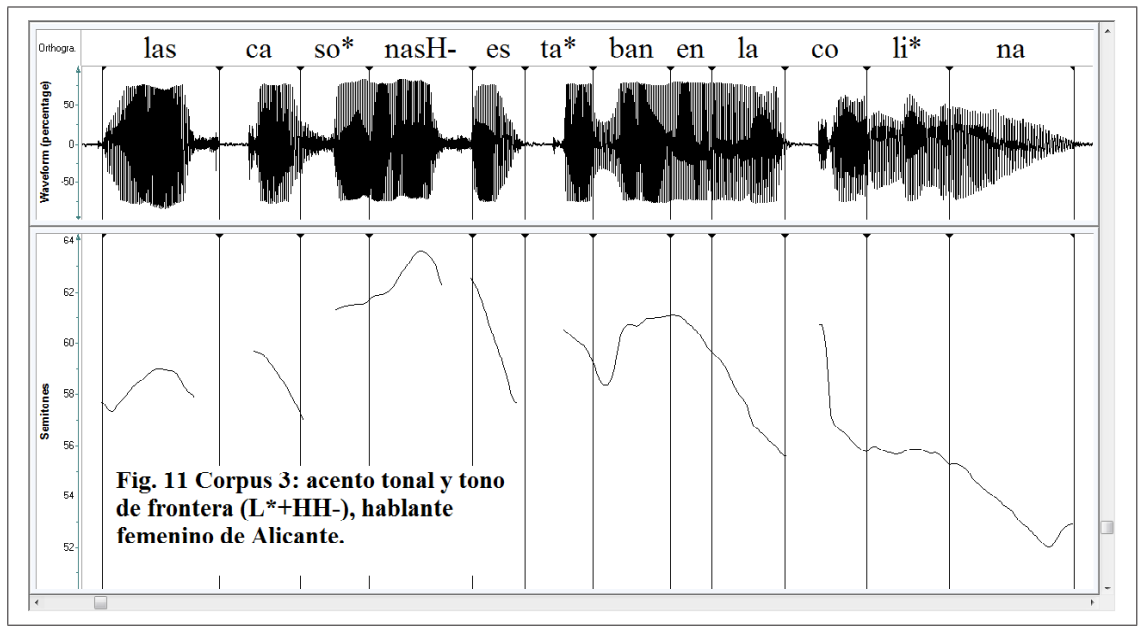

En la figura 11 se presenta una emisión declarativa que es parte del Corpus 3. La emisión es (Las casonas) ip (estaban en la colina) ip IP. Es una producción de un hablante femenino de Alicante. El esquema entonativo es similar a los casos ya presentados: la rama tensiva muestra un movimiento ascendente por el efecto producido por el tono de frontera H-; en la rama distensiva se observa un movimiento descendente. El acento tonal se realiza con un pico retrasado $\left(\mathrm{L}^{*}\right)$; el tono $\mathrm{H}$ se produce en el desarrollo de la sílaba postónica, el tono de frontera se incrusta en la sílaba postónica. Este dialecto, como la mayoría de los dialectos del español, presenta picos retrasados sobre la duración de la sílaba postónica.

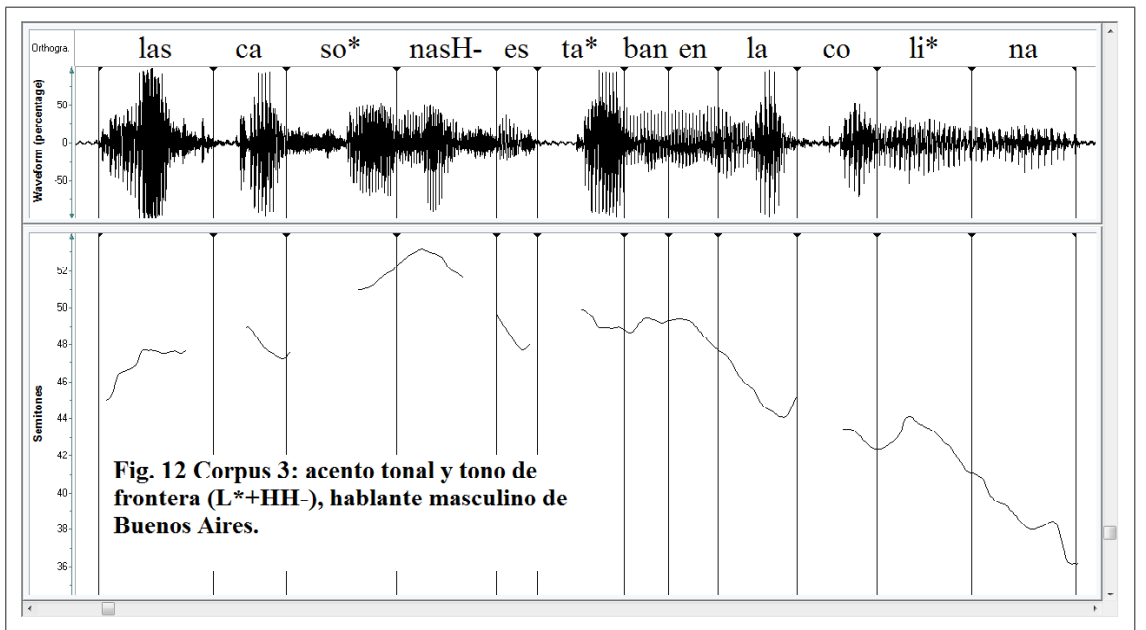


En la Fig. 12 se puede observar la emisión declarativa -(las casonas) ip (estaban en la colina) - ip IP, es una producción emitida por un hablante masculino de Buenos Aires. El dialecto ofrece unos resultados inversos a los de la mayoría de dialectos del español, es decir, el dialecto de Buenos Aires no presenta picos retrasados, la sílaba acentuada es $\mathrm{H}^{*}$ y el tono periférico es, generalmente, L. Puede compararse, por ejemplo, la diferente taxonomía tonal en el acento tonal (estaban), en ambas producciones. El hablante de Buenos Aires produce una sílaba acentuada asociada a un tono $\mathrm{H}^{*}$, es lo opuesto a lo producido en el español de Alicante. Sin embargo, los acentos tonales en la prótasis (en la rama tensiva) son similares en ambos dialectos: son $\mathrm{L}^{*}+\mathrm{H}$. El tono de frontera provoca un acento con un pico retrasado en la sílaba postónica (ver el acento tonal quinto en la figura 1). Las producciones en el dialecto de Buenos Aires confirman la asociación fonológica secundaria en el nivel de la ip, un nivel jerárquico superior a la sílaba acentuada. En el nivel jerárquico de la sílaba acentuada se produce la asociación primaria.

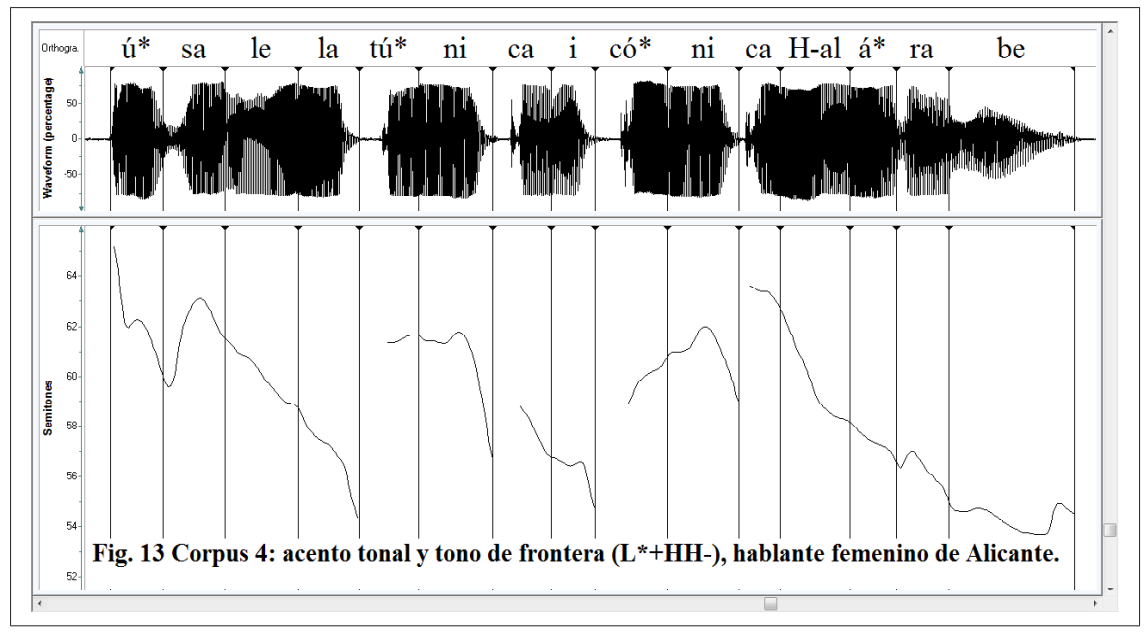

En la figura 13 se observa la emisión -(úsale la túnica icónica) ip (al árabe) ip IP- emitida por un hablante femenino de Alicante, un dialecto con acentos tonales de pico retrasado (Corpus 4). La reestructuración del hablante propone dos ip dentro de la frase mayor. La prótasis presenta un ascenso provocado por el tono de frontera. Los acentos tonales en palabras proparoxítonas (los dos primeros acentos tonales) muestran un efecto tonal diferente al acento tonal final (también en un ítem proparoxítono). Este último presenta una asociación primaria en la sílaba acentuada $\left(\mathrm{L}^{*}\right)$ y un tono periférico $\mathrm{H}$. La asociación secundaria se produce en un nivel jerárquico superior, la ip. El tono de frontera se incrusta en las dos sílabas postónicas, con un movimiento ascendente. En suma, un acento tonal L*+HH-. 


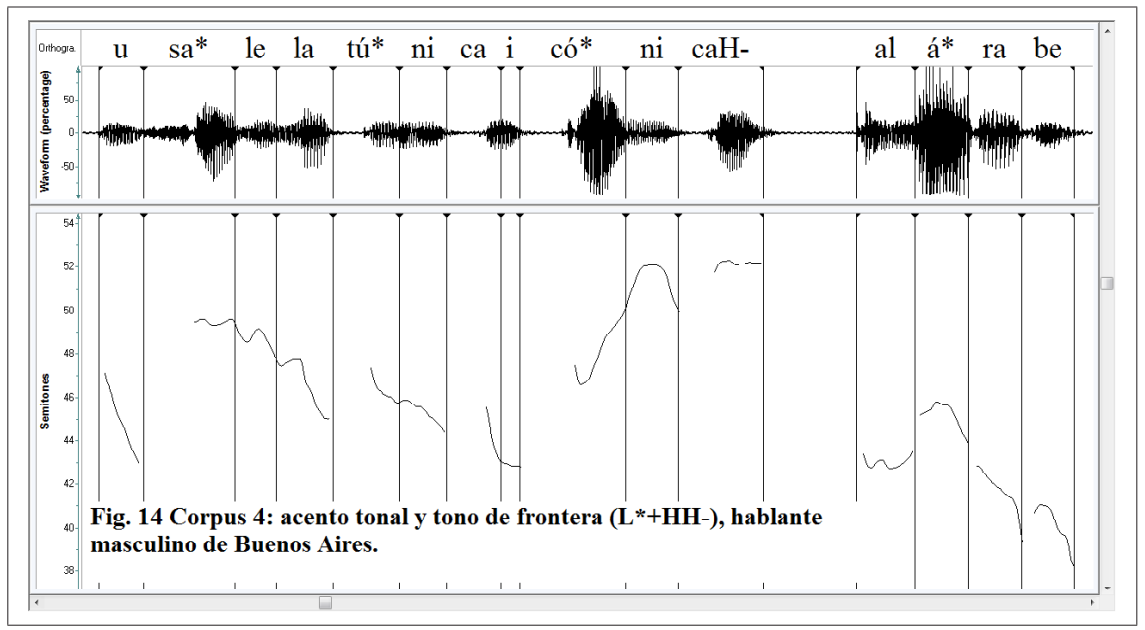

En la figura 14 se consignan las ip que constituyen la emisión declarativa -(usale la túnica icónica) ip (al árabe) ip IP] (Corpus 4). La emisión es una producción de un hablante masculino de Buenos Aires. La reestructuración del este hablante sugiere dos ip dentro de la frase entonativa mayor IP, que es la totalidad de la declarativa. El primer acento tonal de la prótasis (usale) se actualiza en una palabra paroxítona, según el dialecto de Buenos Aires. Los acentos tonales no presentan picos retrasados, excepto en el acento tonal final antes del tono de frontera $\mathrm{H}-$. Este caso confirma la asociación fonológica primaria en $\mathrm{L}^{*}$ y la asociación fonológica secundaria (en el nivel jerárquico superior, la ip) entre el tono de la sílaba postónica y el tono de frontera $\mathrm{H}_{-}$. En síntesis, el acento tonal es $\mathrm{L}^{*}+\mathrm{HH}-$.

\section{Conclusión y Discusión}

Los acentos tonales sufren la influencia de los tonos de frontera. Pamies Bertrán 2007 corrobora también que el pico tonal está asociado con la frontera de los sintagmas, en un sentido amplio, las ip. Esa influencia es relativa al tipo de palabra que recibe el acento tonal. Los acentos tonales dentro de palabras paroxítonas y proparoxítonas presentan una taxonomía $\mathrm{L}^{*+\mathrm{H}}$ (ver el acento tonal quinto en la figura 1). La sílaba acentuada se asocia al tono como $L^{*}$, el tono periférico y en la sílaba postónica, se asocia con un nivel jerárquico superior, el tono de frontera de la ip $(\mathrm{H}-)$. Ese tono de frontera se incrusta también en la sílaba postónica. En el caso de acentos tonales en palabras proparoxítonas el movimiento ascendente de ese tono de frontera continúa en la segunda sílaba postónica. Este fenómeno entonativo podría explicarse por la tendencia de los dialectos del español 
a presentar acentos tonales con pico retrasado. Sin embargo, en un dialecto antípoda a la mayoría de los dialectos españoles, el español de Buenos Aires, también se produce. El español de Buenos Aires no presenta casos de acentos tonales con pico retrasado. Esta tendencia se produce de manera diferente en los acentos tonales dentro de palabras oxítonas. Como es obvio, la presencia del acento en la última sílaba de la palabra produce una doble asociación (primaria y secundaria) en la sílaba acentuada. El fenómeno se registra en una serie de trabajos sobre la influencia del tipo de palabra en la posición del pico acentual. Estebas Vilaplana 2006 indica la importancia de la asociación del pico tonal con el límite de la palabra. Asimismo, señala la importancia del espacio fonético para situar ese pico tonal: a mayor distancia (palabras proparoxítonas y paroxítonas), mayor es el retardo del pico; a menor distancia (palabras oxítonas), menor es el retardo del pico tonal. Sin embargo, en el español de Buenos Aires (un dialecto sin pico retrasado), los acentos tonales prenucleares presentan picos en la sílaba acentuada, sin tener en cuenta la menor o la mayor distancia fonética (ítems oxítonos opuestos a ítems proparoxítonos y paroxítonos). En cambio, este fenómeno se registra en el contexto de la ip y debido a la asociación secundaria producida en ese nivel jerárquico superior. Es evidente que el dialecto de Buenos Aires es la clave para dilucidar este problema. Asimismo, Toledo y Astruc 2008 encuentran resultados similares en corpus leídos por hablantes de Santiago de Chile. Este dialecto comparte con el español de Buenos Aires la tendencia a producir acentos tonales prenucleares con el pico dentro de la sílaba acentuada (Ortiz-Lira 2003). Este dialecto se opone a la mayoría de los dialectos del español.

Un segundo problema es el contraste fonológico de los acentos tonales. Las tres frases ip comunican una realidad pragmática similar: la continuidad semántica de la emisión. La rama tensiva es continuativa, la rama distensiva es conclusiva, es decir, la prótasis opuesta a la apódosis. Sin embargo, la taxonomía es diferente según el tipo de palabra en que se actualiza el acento tonal: $\mathrm{L}^{*}+\mathrm{H}$ (proparoxítonos y paroxítonos) y $\mathrm{L}+\mathrm{H}^{*}$ (oxítonos) (ver los acentos tonales quinto y tercero en la figura 1 , respectivamente).

En síntesis, los acentos prenucleares en emisiones de foco ancho $\left(\mathrm{L}^{*}+\mathrm{H}\right)$ en contraste con el acento nuclear o el acento tonal en foco contrastivo $\left(\mathrm{L}+\mathrm{H}^{*}\right)$ propuesto por el modelo métrico y autosegmental (Sosa 1999; Face 2001; Face 2006) debería revisarse exhaustivamente a la luz de los resultados obtenidos en esta investigación.

\section{REFERENCIAS BIBLIOGRÁFICAS}

Barjam, J. (2004): The intonational phonology of Porteño Spanish,. Los Ángeles, University of California at Los Angeles. 
Beckman, M. E., Díaz-Campos, M., Tevis Mc Gory, J. y Morgan, T. A. (2002): "Intonation across Spanish, in the tones and break indices framework», Probus 14, pp. 9-36.

- Hirschberg, J. y Shattuck-Hufnagel, S. (2005): «The original ToBI system and the evolution of the ToBI framework», en Jun, S. A. (ed.), s. p., www.ling.ohio-state.edu/ tobi/ JunBook /Beck HirschShattuckToBI.pdf.

Colantoni, L. (2005): «Peak alignment of pre-nuclear and nuclear accents in Argentine Spanish», $2^{\text {nd }}$ Spanish ToBI workshop, 22 de junio de 2005, en www. seneca.uab.es/ filologia catalana / papi/tobi/Colantoni.ppt.

Colantoni, L. y Gurlekian, J. (2004): «Early peak alignment and deep fall in Buenos Aires broad focus declaratives», 9th conference on laboratory phonology, Department of Linguistics, University of Illinois at Urbana-Champaign, 24-25 de junio de 2004, en www.linguist.jussieu.fr / marandin /pdf/ cours2.pdf.

Estebas Vilaplana, E. (2006): «Word edge tones in Spanish prenuclear accents», Estudios de Fonética Experimental 15, pp. 11-42.

— 2007: «The phonological status of English and Spanish prenuclear F0 peaks», Atlantis 29, 2, pp. 39-57.

- y Prieto, P. (2007): «Production and perception of word-edge tones in Catalan and Spanish», en González González, M., Fernández Rei, E. y González Rei, B. (eds.), III Congreso Internacional de Fonética Experimental, pp. 279-290.

FACE, T. (2001): Intonational marking of contrastive focus in Madrid Spanish, Tesis doctoral, Ohio, Ohio State University.

- (2006): «Rethinking Spanish L* + H and L + H*», en Díaz-Campos, M. (ed.), Selected proceedings of the $2^{\text {nd }}$ conference on laboratory approaches to Spanish phonetics and phonology, Somerville, MA, Cascadilla Proceedings Project,pp. 75-84.

- y Prieto, P. (2007): «Rising accents in Castilian Spanish: a revision of SpToBI», Journal of Portuguese Linguistics 5, 2/6, 1, pp. 91-115.

GRICE, M. (1995): The intonation of Palermo Italian: Implications for intonation theory, Tubinga, Niemeyer.

Gussenhoven, C. (2002): «Phonology of intonation. state-of-the-article», Glot International 6, pp. 271-284.

HuAlde, J. I. (2002): «Intonation in Spanish and the other Ibero-Romance languages: overview and status quaestionis», en Wiltshire, C. y Camps, J. (eds.), Romance phonology and variation, selected papers from the $30^{\text {h }}$ linguistic symposium on Romance languages, Ámsterdam, John Benjamins, pp. 101-116.

- (2003): «El modelo métrico y autosegmental», en Prieto, P. (ed.), Teorías de la entonación, Barcelona, Editorial Ariel, pp. 155-184.

Jun, S. A. (2005): «Prosodic Typology», en Jun, S. A. (ed.), Prosodic typology: the phonology of intonation and phrasing, Oxford, Oxford University Press, pp. 430-458, en http:/ / www. linguistics.ucla. edu/people/jun/sun-ah.htm.

LADD, R. (1996): Intonational phonology, Cambridge, Cambridge University Press.

Martínez Celdrán, E. y Fernández Planas, A. (2007): Manual de fonética espanola: articulaciones y sonidos del español, Barcelona, Ariel.

Navarro Tomás, T. (1968): Manual de pronunciación española, Decimocuarta edición, Madrid, C.S.I.C. e Instituto Miguel de Cervantes.

Nibert, H. (2000): Phonetic and phonological evidence for intermediate phrasing in Spanish intonation, Tesis doctoral, Illinois, University of Illinois at Urbana- 
Champaign, Urbana-Champaign.

Ortiz-Lira, H. (2003): «Los acentos tonales en un corpus de español de Santiago de Chile: su distribución y realización», en E. Herrera Z., E. y Martín Butragueño, M. (eds.), La tonía: dimensiones fonéticas y fonológicas, México, El Colegio de México, pp. 303-316.

Pamies Bertrán, A. (2007): «Observaciones sobre la estructura melódica en enunciados declarativos», en González González, M., Fernández Rei, E. y González Rei, B. (eds.), III Congreso Internacional de Fonética Experimental, Santiago de Compostela, Xunta de Galicia, pp. 475-488.

- Fernández Planas, A., Martínez Celdrán, E., Ortega, A. y Amorós, A. C. (2002): «Umbrales tonales en español peninsular», en Díaz García, J. (ed.), Actas del II Congreso de Fonética Experimental, pp. 272-278, Sevilla, Universidad de Sevilla.

Pierrehumbert, J. (1980: The phonology and phonetics of English intonation, Tesis doctoral, Cambridge, Massachusetts Institute of Technology.

- y Beckman, M. (1988): Japanese tone structure, Cambridge, The MIT Press.

Prieto, P. (2003): «Efectos de coarticulación tonal en choques acentuales», en Herrera Z., E. y Martín Butragueño, P. (eds.), La tonía: dimensiones fonéticas y fonológicas, México, El Colegio de México, pp. 187-218.

- (2005): «En torno a la asociación tonal en el modelo métrico-autosegmental. Puntos controvertidos en su aplicación al catalán», Revista Internacional de Lingüistica Iberoamericana (RILI) 6, pp. 9-28.

— (2006): "Phonological phrasing in Spanish», en Colina, S. y Martínez-Gil, F. (eds.), Optimality-theoretic advances in Spanish phonology, Ámsterdam y Filadelfia, John Benjamins, pp. 39-60.

— D'Imperio, M. y Gili Fivela, B. (2005): «Pitch accent alignment in Romance: primary and secondary associations with metrical structure», Language and Speech 48, 4, pp. 359-396.

Quilis, A. (1975): «Las unidades de entonación», Revista Española de Lingüística 5, 2, pp. 261-280.

Sosa, J. M. (1999): La entonación del español: su estructura fónica, variabilidad y dialectología, Madrid, Cátedra.

Toledo, G. (2000): «H en el español de Buenos Aires», Langues et Linguistique 26, pp. 107-127.

- (2006): «Fonología entonativa en discursos de Buenos Aires: asociación fonológica secundaria de $\mathrm{T}^{*}$ frente a $\mathrm{H}$-», Language Design, Journal of Theoretical and Experimental Linguistics 8, pp. 131-152.

- (2007): «Fraseo en español peninsular y modelo autosegmental y métrico: asociación secundaria de $\mathrm{T}^{*}$ frente al tono de frontera de frase entonativa intermedia H-», Estudios Filológicos 42, pp. 227-243.

- (2008): «Fonología prosódica en discursos del corpus CREA: asociación fonológica secundaria de T* frente a H-», Revista de Filología 26, pp. 229-244.

—y Ramírez Verdugo, M. D. (2007): «Spanish phrasing: secondary association of $\mathrm{T}^{*}$ in face of intermediate phrase edge tone H- (laboratory data)», Phonetics and phonology in Iberia (PaPI) 2007, Braga, Portugal.

- y Astruc, L. (2008): «Acentos tonales ascendentes en el español de Santiago de Chile», IV Congreso de Fonética Experimental, Granada, 11-14 febrero de 2008. 


\section{ApÉNDICE}

\section{Corpus 1}

Acentos tonales en palabras paroxítonas ( $\mathrm{SP}+\mathrm{SP}$ en choque tonal ip)

(Amar Roma) ip fue inevitable.

(Buscar cosas) ip es lo que hace siempre.

(Cortar cables) ip es su trabajo.

(Dorar muebles) ip es labor de ebanista.

(Esperar siempre) ip es su historia de amor.

(Focalizar puntos en el espacio) ip es la nueva técnica.

(Ganar notas altas) ip es la obsesión del estudiante.

(Hacer buenas acciones) ip es el deber del cristiano.

(Igualar tantos) ip es el juego.

(Jaquear reinas) ip es su deseo.

\section{Corpus 2}

Acentos tonales en palabras paroxítonas, proparoxítonas (punteado) y oxítonas (subrayado) ( SN + SA O SP ip)

(Espárragos blancos) ip con atún marinado.

(Ajos majos) ip con jamón serrano.

(Rabas a la plancha) ip con arroz moreno.

(Chupin de pescado) ip con patatas al natural.

(Cazuela de merluza) ip con puré salteado.

(Profiterol gigante) ip con salsa de estragón.

(Tarta de limón) ip con nisperos caramelizados.

(Timbal de sémola) ip aromatizado con roquefort.

(Bonito a la sartén) ip perfumado con orégano.

(Codornices asadas) ip en sarcófago.

(Pollo a la tártarara) ip con puré de espinacas.

(Pechugas de pavo) ip (con ensalada de coliflor).

\section{Corpus 3}

Acentos tonales en palabras paroxitonas

(Las casonas) ip estaban en la colina.

(Las manzanas) ip estaban en la mesita.

(Las carrozas) ip estaban en el establo.

(Los tomates) ip estaban en la cocina.

(Los pepinos) ip estaban en el canasto.

(Las cebollas) ip estaban en la sopera.

(Las camisas) ip estaban en el ropero.

(Las bananas ip estaban en el armario. 
Acentos tonales en palabras oxítonas

(La mansión) ip se dibujó sobre el azul.

(El peral) ip se destacó sobre el jardín.

(El portón) ip se decoró sobre el balcón.

(El pimentón) ip se acumuló sobre el jamón.

(El cucharón) ip se desplazó sobre el cajón.

(El cascarón) ip se oscureció sobre el tazón.

(El camión) ip se despeñó sobre Gijón.

(El colchón) ip se destrozó sobre el salón.

Acentos tonales en palabras proparoxítonas

Los verbos en el dialecto de Buenos Aires se emiten como palabras paroxítonas (Pedile) y no como palabras proparoxítonas (Pídele) como en el dialecto peninsular. Es similar para el Corpus 4.

(Pídele el tónico) ip al médico.

(Mándale una prótesis) ip al tártaro.

(Arréglale) ip el péndulo ibérico.

(Explícale) ip los filósofos sórdidos.

(Envíale el currículo) ip a Dámaso.

(Úsale la túnica) ip al árabe.

(Tócale) ip unos cánticos tétricos.

(Cómprate) ip una música rítmica.

Corpus 4

Acentos en palabras paroxítonas (SN + SA ip)

(Las casonas marinas) ip estaban en la colina.

(Las manzanas cocidas) ip estaban en la mesita.

(Las carrozas pintadas) ip estaban en el establo.

(Los tomates pelados) ip estaban en la cocina.

(Los pepinos verdosos) ip estaban en el canasto.

(Las cebollas moradas) ip estaban en la sopera.

(Las camisas planchadas) ip estaban en el ropero.

(Las bananas maduras) ip estaban en el armario.

Acentos tonales en palabras oxítonas $(\mathrm{SP}+\mathrm{SA}$ ip)

(La mansión añil) ip se dibujó sobre el azul.

(El peral final) ip se destacó sobre el jardín.

(El portón azul) ip se decoró sobre el balcón.

(El pimentón morrón) ip se acumuló sobre el jamón.

(El cucharón azul) ip se desplazó sobre el cajón.

(El cascarón marrón) ip se oscureció sobre el tazón.

(El camión marrón) ip se despeñó sobre Gijón.

(El colchón añil) ip se destrozó sobre el salón. 
Acentos tonales en palabras proparoxítonas $(\mathrm{SN}+\mathrm{SA}$ ip)

(Pídele el tónico mágico) ip al médico.

(Mándale una prótesis práctica) ip al tártaro.

(Arréglale) ip el mágico péndulo ibérico.

(Explícale) ip los sórdidos filósofos socráticos.

(Enviale el currículo lúdico) ip a Dámaso.

(Úsale la túnica icónica) ip al árabe.

(Tócale) ip unos bárbaros cánticos tétricos.

(Cómprate) ip una mínima música rítmica. 